\title{
Der geschlechtsspezifische Zugriff auf Lesestrategien - Ergebnisse einer Untersuchung im Rahmen unterrichtsdidaktischer Forschung
}

\author{
Doris Grütz (Weingarten)
}

\begin{abstract}
Do girls read differently from boys? This contribution presents a study on the empirical research in classroom teaching following an overview on the findings from research on reading which concerns above all the motivational and emotional dimensions of reading. A research was carried out on 1450 pupils of the 4th year of primary school to find out which reading strategies normally applied in the classroom lead to a successful text comprehension in girls and which in boys. The pattern of the research is based on the cognitivepsychological, hierarchic model of text understanding according to Dijk/Klintsch (1983), which considers the process of reading comprehension as interactional and constructive. Two important results of the study are that in the case of girls certain types of readers develop, whereas with boys it is above all the silent reading of a text which leads to the best reading results.
\end{abstract}

\section{Geschlechtsspezifische Unterschiede beim Lesen: bisherige Befunde}

Das Lese- und Medienverhalten ist von vielerlei Faktoren abhängig. Neben den Determinanten durch soziale Herkunft bzw. Bildung ist es vor allem das Geschlecht, das den Umgang mit Texten prägt und die Lesemotivation beeinflusst (Franz et al. 1999: 79, 86).

\subsection{Mädchen lesen besser als Jungen}

In der PISA-Studie wurde bekräftigt, was man schon längst durch Beobachtung und durch einige wenige Studien (z.B. Richter/Brügelmann 1994) wusste: Mädchen sind den Jungen im verbalen Bereich überlegen (Stanat/Kunter 2001: 249). In allen OECD-Ländern ist der Geschlechterunterschied im Bereich Lesen konsistent: Mädchen sind in ihren Leseleistungen den Jungen signifikant überlegen. In Deutschland macht das im Durchschnitt knapp eine halbe Kompetenzstufe aus (ebda: 251). Dabei zeigt sich ein umso größerer Unterschied, je höher das Anspruchsniveau der reading literacy ist: Beim Ermitteln von Informationen ist die Differenz in der Leseleistung am geringsten, sie wächst beim textbezogenen Interpretieren und ist beim Reflektieren und Bewerten am größten (ebda: 254f.). Stanat und Kunter erklären 
das schlechte Abschneiden der Jungen beim kritischen und reflektierenden Umgang mit Texten mit den Schwierigkeiten der Jungen,

das Gelesene mit eigenen Erfahrungen, Wissensbeständen und Ideen in Beziehung zu setzen. Um ein tief gehendes Verständnis eines Textes zu erreichen, ist es jedoch notwendig, solche Verknüpfungen zwischen den Inhalten und dem eigenen Vorwissen herzustellen. Nur durch die Einbindung des Gelesenen in vorhandene Strukturen wird es möglich, sich einen Text so zu Eigen zu machen, dass seine Inhalte auch noch zu einem späteren Zeitpunkt im Gedächtnis verfügbar sind und genutzt werden können (ebda: 266).

Eine mangelnde Nutzung von Vor- und Erfahrungswissen dürfte auf den hierarchieniedrigen Kompetenzstufen auch die Fähigkeit zur Informationsermittlung und zur Textinterpretation negativ beeinflussen. Richter und Christmann weisen darauf hin, dass beim Aufbau eines mentalen Modells bereits auf der Satzebene bei der lokalen Kohärenzbildung das Vorwissen bzw. allgemeine Weltwissen zum Tragen kommt, indem z.B. logische Strukturen wie Bedingungen, Grund-Folge, etc. erkannt werden (2002: 30). Durch die Aktivierung des Vorwissens ist es auch möglich, Defizite auf hierarchieniedrigen Verarbeitungsebenen auszugleichen (ebda: 45; 48). Beim Lesen mitzudenken und dadurch sinnerschließend zu lesen, kann über Schwierigkeiten bei der Worterkennung hinweghelfen.

\subsection{Die Textsorte beeinflusst die Leseleistung}

In Bezug auf den Geschlechterunterschied in den Leistungen zwischen literarischen und Informationstexten zeigen sich folgende Befunde. In der IGLU-Studie erzielen Mädchen etwas bessere Werte beim Lesen literarischer Texte. Die Differenz zum Lesen von Informationstexten ist in Deutschland recht gering (vgl. Bos et al. 2003: 114). Auffällig sind für Deutschland die deutlich größeren Abstände im Leseverständnis zwischen den Geschlechtern bei 15-Jährigen in der PISA-Studie: Die Geschlechterdifferenz liegt bei kontinuierlichen Texten weit höher als bei nicht-kontinuierlichen Texten. Differenziert man nach Textsorten, ergibt sich folgendes Bild: "Während bei Erzählungen, Argumentationen sowie Darlegungen recht große Geschlechterunterschiede zu Gunsten der Mädchen zu verzeichnen sind, ist die Differenz bei Tabellen erheblich kleiner und bei Diagrammen/ Grafen, Karten und schematischen Zeichnungen fast völlig verschwunden" (Stanat/Kunter 2001: 255). Z. T. weisen die Jungen hier sogar bessere Leseleistungen auf; allerdings sind diese Werte statistisch nicht signifikant (ebda: 260).

Leseverstehen ist auch abhängig vom Medium. In der Schweizer Studie "Literalität im medialen Umfeld" verringerte sich das Aktivitätsgefälle zwischen Mädchen und Jungen, wenn die Schüler/innen zwischen Buch- und Bildschirmlektüre wählen konnten, wohingegen in "traditionellen Buchumgebungen ... die Leseaktivitäten der Jungen weit hinter jener der Mädchen" zurückblieben (Bertschi-Kaufmann 2002b: 145f.).

Eine Erklärung für die unterschiedlichen Präferenzen und den damit verbundenen Leseleistungen mag Baron-Cohen (2004) geben, der genetische Determinationen für das geschlechterspezifische Verhalten verantwortlich macht. Er schreibt dem weiblichen Gehirn größere Empathiefähigkeit zu, dem männlichen Gehirn stärkere Systemorientierung. 
"Mehrere Studien deuten darauf hin, dass dreijährige Mädchen ihren männlichen Altersgenossen bereits weit voraus sind, wenn es darum geht, die Gedanken und Gefühle anderer Menschen zu deuten, d.h. eine theory of mind anzuwenden. $\mathrm{Zu}$ dieser kognitiven Komponente der Empathie gehört im Wesentlichen, dass man die eigene derzeitige Perspektive vorübergehend außer Acht lässt, der anderen Person einen bestimmten Gemütszustand oder eine bestimmt "Einstellung" zuschreibt und Schlüsse über ihre mutmaßliche innere Verfassung zieht, indem man auch ihre Erfahrungen berücksichtigt" (Baron-Cohen 2004: 46).

Die Fähigkeit von Mädchen und Frauen, sich in andere Personen und Situationen hineinzuversetzen und Vorhersagen über den weiteren Handlungsverlauf zu treffen, weist eine gewisse Stimmigkeit mit der Präferenz für literarische und im allgemeinen kontinuierliche Texte und den guten Leseleistungen bei diesen Textsorten und Textarten auf. Bei den Jungen stellt Baron-Cohen ein besonderes "Systematisierungsvermögen", die Fähigkeit zu einem methodisch-analytischen Vorgehen, fest.

Dieses "zeigt sich in dem Drang, Systeme zu analysieren, zu erforschen oder zu entwickeln. Wer seine Wahrnehmungen in ein System bringen will, versteht intuitiv, wie etwas funktioniert oder durch welche übergreifenden Regeln das Verhalten eines Systems gesteuert wird. Ziel ist, das System zu begreifen und sein Verhalten vorherzusagen oder ein neues zu erfinden. (...) Jungen spielen auch gerne mit Dingen, die klare Funktionen haben. (...) Kurz: Sie sind ganz begeistert von Systemen" (Baron-Cohen 2004: 46f.).

Baron-Cohens Darlegungen stimmen auch mit der Präferenz von Jungen für diskontinuierliche Texte, wie Tabellen, Diagramme, grafische Darstellungen, Karten und schematische Zeichnungen, und mit der Präferenz für Bildschirmtexte überein.

Eine psycho-soziologische Erklärung für geschlechterspezifische Differenzen bei der Wahl der Lektüre findet sich in der kognitiven Theorie der Geschlechtsro1lenidentifikation von Kohlberg (1974), die bei Oerter (1999) rezipiert ist:

Kohlberg (1974) geht davon aus, dass das Kind selbst und nicht in erster Linie der soziale Druck von außen die Geschlechtsro1lenidentifikation vorantreibt. Das Kind erkennt zunächst, dass es zwei Geschlechter gibt und ordnet sich einem der beiden Geschlechter zu. Aus Untersuchungen wissen wir, dass dies bereits zu Beginn der zweiten Lebensjahres geschieht. Sobald diese Zuordnung getroffen ist, wählt das Kind die Verhaltensweisen aus seiner Umwelt aus, die zu seinem Geschlecht passen. Daher imitiert es auch nicht in erster Linie den Vater bzw. die Mutter, sondern Rollenträger, die Geschlechtsrollenmerkmale besonders anschaulich repräsentieren, wie etwa Indianer und Cowboys bzw. Squaw und Prinzessin. Auch die Wahl von Spielsachen folgt nach Kohlberg diesem Muster, denn Kinder bevorzugen häufig auch dann geschlechtsspezifisches Spielzeug, wenn man ihnen gegengeschlechtliches Spielzeug anbietet. Schließlich erkennt das Kind die Geschlechtsrollenidentität, d.h. es weiß um die Unveränderbarkeit des biologischen Geschlechtes. Dieser Werdegang ist mit vielen Untersuchungen im großen und ganzen belegt (Oerter 1999: 46f.).

Oerter schlägt eine Verbindung des biologischen und des konstruktivistischen Ansatzes vor und kommt im Anschluss an Nunner-Winkler (1998) zu dem Schluss, dass "es in der Hauptsache das heranwachsende Kind selbst ist, das sich geschlechtsspezifisch angemessene Literatur auswählt" (Oerter 1999: 47). 
So "erstaunt es nicht", um mit Bonfadelli und Fritz (1993) zu reden, "dass sich in der Zusammensetzung der Lektüre starke geschlechtsspezifische (...) Unterschiede spiegeln. (...) Mädchenbücher und Schicksals- sowie Liebesromane werden von den Mädchen präferiert, während bei Knaben eher das Sachbuch dominiert; Abenteuerbücher hingegen sind beim männlichen wie beim weiblichen Geschlecht gleichermaßen beliebt" (Bonfadelli/Fritz 1993: 54f.).

Um einen Ausgleich zwischen der biologisch-deterministischen und der konstruktivistischen Position ist auch Nickel-Bacon bemüht, die angesichts der unterschiedlichen Lektürepräferenzen von Mädchen und Jungen von unterschiedlichen "Geschmackskulturen" der Geschlechter spricht (2003: 31).

Die Ausführungen zu Vorstellungen von einer biologisch bedingten und einer konstruierten Geschlechterrolle und der damit verbundenen Präferenz für Themen, Textsorten und Medien führen zu den Faktoren Interesse und Motivation als Variablen für das Leseverstehen.

\subsection{Interesse und Motivation beeinflussen die Leseleistung}

In der PISA-Studie wurde deutlich, dass motivationale Faktoren die Leseleistung erheblich beeinflussen können. Besonders in Deutschland klafft ein großer Unterschied zwischen 15jährigen Mädchen und Jungen in Bezug auf die Freude am Lesen: Fast ein Drittel aller Mädchen und sogar über die Hälfte aller Jungen gibt an, nicht zum Vergnügen zu lesen (Stanat/Kunter, 2001: 263). Lesen Mädchen also besser, weil sie lieber lesen und interessierter sind? PISA-Befunde bestätigen dies: Der Geschlechterunterschied zugunsten der Mädchen fällt nur noch sehr gering aus, wenn Mädchen und Jungen ähnliches Interesse am Lesen haben, er verschwindet ganz bei gleicher Freude am Lesen (ebda: 265).

In einer Studie von van Kraayenoord und Schneider (1999) wird deutlich, dass sich die Interessen von Kindern in der Grundschule kaum unterscheiden. Beide Geschlechter interessieren sich gleichermaßen für Abenteuerromane, Jungs etwas mehr für Comics und informierende Texte, Mädchen mehr für Problembücher (s. auch Bertschi-Kaufmann 2002a: 153; 2002b: 145; Bischoff/Heidtmann 2002b: 28; Richter/Plath 2002: 48).

In der IGLU-Studie zeigt sich denn auch bei Weitem kein so großer Unterschied zwischen den Geschlechtern wie in der PISA-Studie (Bos et al. 2003: 114ff.). Die Autor/innen beurteilen das Ergebnis zwischen Mädchen und Jungen sogar als "ausgeglichen", auch wenn die Mädchen im Leseverständnis bessere Werte als die Jungen aufweisen (Mädchen 545, Jungen 533 Punkte Mittelwert). Ausschlaggebend ist bei der Interpretation der internationale Vergleich, bei dem die Differenz zwischen den Geschlechtern in Deutschland relativ gering ausfällt und sogar über dem Durchschnitt der EU-Länder (vor dem1. Mai 2004) ${ }^{1}$ liegt. Auch bleibt der Unterschied zwischen den Textsorten gering: Zwar lesen Mädchen literarische Texte besser als die Jungen, doch beträgt der Vorsprung nur 14 Punkte; bei Sachtexten liegt der Vorsprung bei nur 10 Punkten. Hier zeigt sich schon, wenn auch noch nicht so deutlich

\footnotetext{
${ }^{1}$ Die Vergleichsgruppen bei der IGLU-Studie sind in Bos et. al (eds.) 2003: 9 beschrieben.
} 
wie bei den 15-Jährigen der PISA-Studie, die Vorliebe der Jungen für Sachtexte, die sich in entsprechend besseren Leseleistungen bei dieser Textsorte niederschlägt.

Das thematische Interesse bleibt im Laufe der Lesesozialisation stabil. Bei Jugendlichen und jungen Erwachsenen zwischen 14 und 29 Jahren konstatiert Köcher eine deutliche Vorliebe der Frauen für Unterhaltungsliteratur und der Männer für Fachbücher (Köcher 1993: 307f.). Interessant ist dabei die emotionale Beziehung der Geschlechter zum Informationslesen:

"Durch das ausgeprägte Ausrichten auf das Unterhaltungslesen erleben Frauen trotz ihrer grundsätzlich größeren Lesefreude Pflichtlektüre teilweise belastender als Männer" (ebda). "Die Beziehung von Männern zu Büchern ist kühler, zweckorientierter als bei Frauen. Mehr als Frauen tendieren Männer dazu, Bücher nur dann zu lesen, wenn sie das Thema besonders interessiert; mehr als Frauen nutzen Männer Bücher auch als Nachschlagewerke, um bestimmte Fragen zu klären. Männer neigen auch überdurchschnittlich dazu, Bücher nicht zu Ende oder quer zu lesen" (ebda: 308$)$.

Aus dieser wie aus anderen Untersuchungen ergibt sich, dass nicht nur das thematische Interesse, sondern auch die Funktion des Lesens bei den Geschlechtern unterschiedlich sind. Eine neuere Untersuchung von Bischoff und Heidtmann (2002) relativiert allerdings dieses traditionelle Bild. Die Autor/innen stellen fest:

"Jungen aller Altersstufen nutzen erzählende Literatur - wie andere Medien auch - immer stärker ausschließlich unterhaltungs- und erlebnisorientiert. Der Aspekt des informatorischen Lesens, den frühere Erhebungen bei männlichen Lesern als zentral nachgewiesen haben, verliert an Bedeutung. Um zu lernen, um sich über ein Thema zu informieren, lesen gerade noch $11 \%$ der Befragten. Um Spaß zu haben, um sich mit spannender Lektüre zu unterhalten, um sich die Zeit zu vertreiben, um abzuschalten und sich zu entspannen, lesen $40 \%$ der Jungen" (Bischoff/Heidtmann 2002b: 29).

Kann bei Unterschieden in der schulischen Leseleistung zu Gunsten der Mädchen auf eine ungleiche Berücksichtigung der motivationalen Faktoren zu Lasten der Jungen geschlossen werden? Das thematische Interesse und die Freude am Lesen dürften in der Grundschule bei beiden Geschlechtern angesprochen und befriedigt werden (Franz et al. 1999: 83). Weitgehend unberücksichtigt bleiben jedoch die stärkeren Interessen der Jungen an Bildschirmtexten. Dies wurde durch die IGLU-Studie deutlich:

"Der Ausstattung mit Computern kommt von Seiten der Schulleitungen in Deutschland besondere Aufmerksamkeit zu: Auf die Frage, inwieweit der Leseunterricht durch den Mangel in verschiedenen Bereichen negativ beeinflusst wird, nennen die Schulleiter an erster Stelle fehlende Computerfachleute (für 69\% der Schüler wird hier ein Mangel erlebt), an zweiter Stelle den Mangel an Computerprogrammen (51\%) und an dritter Stelle den Mangel an Computern für Unterrichtszwecke (44\%). Erst mit großem Abstand folgen Bücher in der Bibliothek (25\%), audiovisuelle Mittel (22\%) und Lehrpersonal (20\%)" (Bos et al. 2003: 36$38)$. 


\subsection{Bestimmt das Alter den Geschlechterunterschied beim Lesen?}

Ein Vergleich der Befunde zur Leseleistung zwischen der IGLU-Studie und der PISA-Studie zeigt, dass sich die Geschlechterdifferenz mit zunehmendem Alter erhöht. Eine deutliche Differenzierung ergibt sich auch in der Leseintensität: Richter und Plath stellen in ihrer Studie von 2001 einen ersten Leseknick bei beiden Geschlechtern bereits nach der 2. Klasse fest. Davon sind die Jungen stärker betroffen als die Mädchen. Eine Erklärungsmöglichkeit bietet der psychoanalytische Ansatz, den Garbe (2003a und 2003b) nahe legt: Jungen wenden sich mit Beginn des Bewusstwerdens über die eigene Geschlechterrolle vom weiblich konnotierten Lesen ab. Dies beginnt in der Familie, wo Frauen häufig die Vorlesenden sind, und setzt sich in der Grundschule fort. Aus der IGLU-Studie wurde deutlich, dass

"wie in den meisten teilnehmenden Ländern ... auch in Deutschland die meisten Schülerinnen und Schüler an Grundschulen von Frauen unterrichtet (werden), nämlich 82\%. In der Vergleichsgruppe 1 (teilnehmende Lände aus der EU: Deutschland, England, Frankreich, Griechenland, Italien, Niederlande, Schottland, Schweden) sind es $77 \%$ aller Grundschülerinnen und -schüler" (Bos et al. 2003: 42).

Bischoff und Heidtmann zeichnen für das Grundschulalter allerdings ein weniger düsteres Bild von der Leseintensität der Jungen:

"Gegen Ende des Grundschulalters nutzen Jungen am zeitaufwändigsten und intensivsten erzählende Literatur. Die Anzahl der pro Monat gelesenen Bücher erreicht im Alter zwischen 10 und 13 Jahren mit ein bis fünf Titeln pro Monat ihren Höhepunkt." (Bischoff/Heidtmann 2002b: 29).

Einigkeit besteht jedoch darin, dass die Leseintensität nach dem Grundschulalter abnimmt und sich dieser Prozess bei den Jungen im Laufe der Jahre fortsetzt: Harmgarth (1999) sowie Bischoff und Heidtmann (2002b: 29) konstatieren einen "Leseknick" bei Jungen in der Pubertät. In der Studie der Bertelsmann Stiftung von 1993 zeigten sich in der Altersgruppe von 16 - 29 Jahren die Frauen noch als intensive Leserinnen mit $36 \%$, im Gegensatz zu den Männern mit 27 \% (Köcher 1993: 302; 305). Erst ab dem 30. Lebensjahr gab es in der Untersuchung einen starken Leseknick, der die Frauen sogar unter das Leseintensitätsniveau der Männer herabdrückte: Frauen: $21 \%$, Männer 24\% (ebda.). Die Gründe wurden in der zeitlichen Beanspruchung durch familiäre und berufliche Aufgaben vermutet.

Insgesamt ist beim männlichen Geschlecht eine kontinuierliche Abnahme der Leseintensität, beim weiblichen Geschlecht eine weitgehende Stabilität der Leseintensität (bis zum 30 . Lebensjahr) zu verzeichnen. Dieses Leseverhalten hängt mit der unterschiedlichen Funktion des Lesens bei den Geschlechtern zusammen. Auf das eher informatorische, zweckorientierte Lesen des männlichen Geschlechts wurde schon im vorigen Kapitel hingewiesen. Anders bei Mädchen und Frauen:

"Für Frauen ist Lesen weitaus mehr als für Männer ein ganzheitliches Erlebnis, das nicht nur rational verarbeitet wird, sondern auch emotional große Bedeutung hat. Nicht zu übersehen ist auch, dass weibliches Lesen teilweise eskapistische Formen annimmt und als Gegenwelt zu der eigenen erlebten Realität empfunden wird. Frauen tendieren weniger als Männer dazu, bei ihrer Lektüre scharf nach bestimmten Zielen und Interessen zu selektieren" (Köcher 1993:220). 
Die Befunde der 1992 durchgeführten Studie der Bertelsmann Stiftung, dass Männer eher Fachliteratur lesen und Literatur zielorientierter rezipieren, Frauen sich hingegen mehr der unterhaltenden Literatur widmen, wurde in der Studie der Stiftung Lesen aus dem Jahr 2000 bestätigt. In der Studie der Stiftung Lesen wurde jedoch ein Anstieg an weiterbildendem Lesen und an Informationslesen bei beiden Geschlechtern festgestellt (2001:14f). Dieser Befund dürfte auf die veränderten sozioökonomischen und kulturellen Bedingungen innerhalb des knappen Jahrzehnts, das zwischen den beiden Studien liegt, zurückzuführen sein.

Nicht das Alter per se bestimmt das Leseverhalten, sondern es werden in erster Linie Fragen der Motivation, des Interesses, des Entwicklungsstandes, der familialen und schulischen Lesesozialisation, der Bildung und auch der verfügbaren Zeit wirksam. Ein weiterer wichtiger Faktor liegt in einem Bereich, der als Schnittmenge zwischen Selbststeuerung und Einwirken des sozialen Umfeldes gilt: dem Selbstkonzept.

\subsection{Selbstkonzept}

Leseleistung hängt von der Leseintensität $\mathrm{ab}$, die Leseintensität wiederum von der Motivation und dem Interesse. In der Grundschule ist die Leseleistung von Mädchen besser als die von Jungen. In der oben zitierten Studie von Kraayenoord und Schneider (1999) wird aber deutlich, dass sich die Interessen von Kindern in der Grundschule kaum unterscheiden. Beide Geschlechter interessieren sich gleichermaßen für Abenteuerromane, Jungs etwas mehr für informierende Texte, Mädchen mehr für Problembücher (Richter/Plath 2002: 48). Kann dann die Leseleistung so stark vom Interesse abhängen?

In der PISA-Studie bestätigt Japan überdeutlich, dass diese Korrelation nicht so ohne weiteres gesehen werden darf: Die Leseleistung von 15-jährigen japanischen Schüler/innen liegt signifikant über dem OECD-Mittlelwert, die deutscher Schüler/innen signifikant darunter (Stanat et al. 2003: 52). Beide Gruppen geben jedoch ähnlich oft an, nicht zum Vergnügen zu lesen (Artelt et al. 2001: 114). Extrinsische Motivation und ein Selbstkonzept, das auf eine Erfüllung schulischer Normen gerichtet ist, dürften hier die guten Leseleistungen bedingen.

Von einem unterschiedlichen Selbstkonzept kann auch zwischen Mädchen und Jungen ausgegangen werden. Aus Interaktionsanalysen von schulischen Kleingruppen wurde deutlich, dass Mädchen viel stärker als Jungen an der Lösung von Aufgaben interessiert sind, zielorientiert arbeiten und auf die Erfüllung der Schulnorm gerichtet sind. "Mädchen sind sachbezogen, aufmerksam, kooperativ, die Knaben sind desinteressiert, unruhig, laut" (Rüegg 1995: 174). Etliche Untersuchungen, z.B. von van Alphen (1996) und Fuchs (2001) bestätigen diese Beobachtung. Für Mädchen haben Gespräche eine andere Funktion als für Jungen. Dies zeigt sich nicht nur bei Gesprächen in der Schule, sondern auch in Freundesgruppen. So kommt Fuchs zu folgender Beurteilung des kommunikativen Verhaltens der Geschlechter in Peer-groups:

Jungen machen andere Jungen lächerlich, drohen und geben an, verweigern die Befolgung von Aufforderungen und Befehlen und versuchen, Wortgefechte zu gewinnen. Sie lernen, Sprache 
als verbale Waffe zu benützen, mit der sie ihren eigenen Anspruch auf Dominanz stützen können, und mit der sie die Ansprüche anderer Jungen zurückweisen können. (...)

Mädchen etablieren in ihren Gruppen enge und hochemotionale Freundschaften, in denen Gespräche sehr wichtig sind. Es herrscht die Vorstellung der grundsätzlichen Gleichheit, so dass Unterschiede zwischen ihnen in der relativen Enge der Beziehung liegen und weniger in der relativen Dominanz oder dem relativen Status. Sie bevorzugen gemeinsames Geschichtenerzählen, und benützen weniger direkte Handlungsanweisungen als vielmehr begründete und inklusive Aussagen". (...) Bei Konflikten lernen Mädchen, "andere zu unterstützen, ihre Rederechte zu beachten, ihre eigene Rede mit der anderer Mädchen zu verknüpfen und in ihren Spielen auch sprachlich zu kooperieren" (Fuchs 2001: 74ff.).

Mädchen "üben" in ihren Freundesgruppen zu reden und profitieren davon auch in der Schule. Wie wichtig Freundschaft an sich für Lese- und Schreibfähigkeiten sind, darauf verweist Oerter (1999). Er zitiert eine Untersuchung von Pellegrini et al. (1997), wonach die Konfliktlösung unter Freunden zu einem differenzierten sprachlichen Verhalten und einer erhöhten sprachlichen Bewusstheit führt: "Sowohl die mündlichen als auch die schriftlichen Leistungen waren nach einem Jahr bei den Kindern, die einen engen Freund hatten, signifikant besser" (Oerter 1999: 34).

Kompetitives und auf Selbstdarstellung orientiertes Verhalten gehört trotz offensichtlicher schulischer und persönlicher Nachteile zu dem gängigen Rollenbild von Jungen, das immer wieder erprobt wird.

"Stör-Kommunikation und sogar das Abweichen von schulischen Normen mag unter Jungen eine Art von "doing gender" darstellen. Sobald sie Mädchen als leistungsorientiert und unterrichtszentriert erfahren, erleben sie dies wohl als feminin. Es gehört dann zu ihrer Identitätspolitik, sich davon abzusetzen" (Kotthoff 2003: 87).

Lernwillige Jungen müssen sich erst gegen ihre Mitschüler durchsetzen, bevor sie sich am Unterricht beteiligen können. Hentschel (2000) und Kotthoff (2003) weisen darauf hin, dass dieses Leitbild der Männlichkeit zum "Leiden an der Männlichkeit" (Hentschel 2000: 7) führt. Die zu erfüllenden Rollenmuster machen Jungen und Männer seelisch und körperlich krank. Doch dies findet oft zu wenig Beachtung.

\subsection{Zusammenfassung}

Es wurden verschiedene Einflussfaktoren auf das Leseverstehen beschrieben: Die generelle Überlegenheit von Mädchen in der Leseleistung lässt das Geschlecht an sich als wichtigen Faktor für die Voraussage von Leseleistung gelten. Dies relativiert sich jedoch, wenn unterschiedliche Themen, Textsorten und Medien bei der Datenauswertung berücksichtigt werden. In Abhängigkeit von Interesse und Motivation gleichen sich die Leseleistungen von Mädchen und Jungen an. Damit zusammenhängende Faktoren sind das Alter der Kinder und Jugendlichen sowie ihr Selbstkonzept. Während bei den Jungen die Leseleistungen ab Ende der Grundschulzeit sinken, bleiben sie bei den Mädchen stabil. Mädchen sind aufgrund ihrer Sozialisation eher gewillt, die Schulnorm zu erfüllen. 
Diese den emotionalen und motivationalen Dimensionen des Leseverstehens zuzuschreibenden Faktoren können Leseleistungen jedoch nur zum Teil erklären. Hinzu kommt, was das schulische Lesen anbelangt, ein weiterer Aspekt: die Anwendung von Lesestrategien. In der Studie von Belgrad/Grütz/Pfaff (2003) wurde in der Grundschule 4. Klasse getestet, welche der im Unterricht gängigen Lesestrategien tatsächlich zum Erfolg führen und welche dieser Strategien bei den Mädchen, welche bei den Jungen ein besseres Textverständnis hervorrufen.

\section{Die Lesestrategie beeinflusst das Textverständnis}

In der Studie von Belgrad/Grütz/Pfaff (2003) geht es um die Frage, wie gelesen wird, welche Strategien angewandt werden, um Texte zu verstehen. Vor dem theoretischen Hintergrund eines konstruktivistischen Lesebegriffs wurde untersucht, welche Zugriffsweisen Mädchen und Jungen auf Lesestrategien haben.

\subsection{Leseverstehen - ein interaktionaler, konstruktiver Prozess}

Der Verstehensprozess ist durch ein ständiges Interagieren zwischen den Informationen aus dem Text (Bottom-up-Prozess) und dem Input seitens des Lesers (Top-down-Prozess) gekennzeichnet (Rumelhart 1994; Christmann/Groeben, 2002).

"Der Text bezieht sich auf ein bestimmtes Thema und beinhaltet spezifische Sprach- und Strukturelemente, welche die Lesbarkeit beeinflussen. Bedeutsam sind auch die Textsorten bzw. das vom Verfasser verfolgte Ziel - will der Verfasser unterhalten, überzeugen oder informieren"

(Bos et al. 2003: 72).

Der Leser bringt eine Reihe von Voraussetzungen mit, die das Niveau des Leseverständnisses beeinflussen. Hierzu zählen Faktoren, die dem Leseprozess vorausgehen bzw. ihn begleiten, wie Leseerfahrung, Motivation (Leseabsicht, Interesse, Selbstwirksamkeit) und kognitive Voraussetzungen (Aufmerksamkeit, Gedächtnis, Wortschatz, analytische Kompetenz). Faktoren, die das aktuelle Lesen beeinflussen, sind die basale Dekodierfähigkeit (Sicherheit und Schnelligkeit der Wortidentifikation) und das Wissen des Lesers (allgemeines Weltwissen, textspezifisches Vorwissen und metakognitive Strategien).

Die Dekodierfähigkeit auf Wort- und Satzebene sowie das Vorwissen des Lesers bilden die beiden Pole im kognitionspsychologischen, hierarchischen Situationsmodell des Leseverstehens von van Dijk und Kintsch (1983). Textverstehen gestaltet sich als interaktiver und konstruktiver informationsverarbeitender Prozess: Die im Text enthaltenen Informationen verbindet die Leserin/der Leser aktiv mit ihrem/seinem Vor- und Weltwissen und konstruiert damit eine individuelle, mentale Repräsentation des Gelesenen.

Manche Modelle zum Textverstehen erachten eher die hierarchieniedrigen Prozesse der Worterkennung als ausschlaggebend: Nach Perfettis Theorie der verbalen Effizienz (1985) ist $\mathrm{zu}$ vermuten, dass ein schneller und sicherer Zugriff auf die Wortbedeutung auch auf hierarchiehöhere Verarbeitungsprozesse positiv wirkt und gute Leser/innen ausmacht. 
Andere Modelle sehen die hierarchiehohen Verarbeitungsprozesse als für das Textverständnis ausschlaggebend an. Nach Oakhill und Garnham (1988) kann echtes Textverständnis nur über adäquates inhaltliches Vorwissen erlangt werden. Gute Leser/innen besitzen die Fähigkeit, textseitige Informationen mit verstehensrelevanten Inferenzen $\mathrm{zu}$ ergänzen sowie die übergreifende Struktur des Textes zu erkennen und wesentliche Aussagen des Textes zu erfassen. Nach der Schematheorie von Spiro (1980) hält das Alltags-, Welt- und Sachwissen Schemata und Skripts zur Einordnung von Informationen bereit: Schemata als Repräsentation von Wissen über typische Zusammenhänge von Bereichen der Welt/Realität, Skripts als spezielle Form von Schemata, die Wissen über routinisierte Verhaltens- und Ereignisabläufe abbilden. Yekovich und Walker (1987) konnten nachweisen, dass das Vorwissen den Leseprozess weitaus stärker beeinflusst als die semantisch-syntaktischen Informationen aus dem Text. In Untersuchungen zur Rolle des Vorwissens (z.B. Spiro 1980; Stanovich 1980; Yekovich/Walker 1987) hat sich auch gezeigt, dass Verarbeitungsprozesse auf höherer Ebene Defizite in hierarchieniedrigen Verarbeitungsprozessen ausgleichen können (vgl. Richter/Christmann 2002: 45, 48).

Während hierarchieniedrige Prozesse eher automatisch ablaufen, gehen hierarchiehohe Verarbeitungsprozesse des Lesens strategisch-zielbezogen vonstatten (ebda: 31). Bei der Frage nach der Anwendung und Wirksamkeit von Lesestrategien rückt somit der Faktor Wissen in den Vordergrund. Leser, die effektive Lesestrategien kennen und das Gelesene verarbeiten können, konstruieren Bedeutung aktiv (Bos et al., 2003: 71f). Dabei werden die verschiedenen Lesestrategien unbewusst oder bewusst angewendet.

\subsection{Lesestrategien}

Der Hinweis auf den unbewussten bzw. bewussten Einsatz von Lesestrategien führt zu dem situativen Kontext, in dem Lesestrategien gezielte Anwendung finden: zum Unterricht in der Schule. Die Fragestellung in unserer Studie zur Wirksamkeit von Lesestrategien lautete: Welche Abfolge von Strategien zur Informationsaufnahme aus Sachtexten sind am erfolgreichsten (cf. ausführlich in Belgrad/Grütz/Pfaff 2004) ? Ziel war es, einem kontinuierlichen Sachtext Informationen zu entnehmen. Dabei kam es nur auf das Verstehen der Informationen, nicht auf das Behalten an. Es wurden Strategien getestet, die im Unterricht der 4. Klasse Grundschule als gängige Verfahren der Texterschließung gelten.

Wir gingen davon aus, dass im Unterricht Texte zweimal rezipiert werden. Für den 1. Rezeptionsdurchgang wählten wir Strategien, die sich für die Textbegegnung eignen, für den 2. Rezeptionsdurchgang wählten wir Strategien, die auf das vertiefte Textverstehen abzielen. Die Strategien des 1. und 2. Rezeptionsdurchgangs wurden miteinander kombiniert, so dass wir Methodenkombinationen testeten (s. Forschungsdesign Abb. 1).

Für den ersten Rezeptionsdurchgang wählten wir folgende Methoden:

(1) Stilllesen: Die Schüler/innen lesen den Text still durch

(2) Vorlesen: Die Lehrkraft liest den Text vor

(3) Aktivieren von Vorwissen: Vor der Textbegegnung wird im Unterrichtsgespräch Vorwissen aktiviert. 
Die Verfahren (1) und (2) entsprechen den hierarchieniedrigen Ebenen des Textverstehen: Es werden die visuelle und die auditive Dekodierfähigkeit angesprochen. Das Verfahren (3), die Aktivierung von Vorwissen, betrifft den hierarchiehohen Verarbeitungsprozess der Anwendung metakognitiver Strategien.

Für den zweiten Rezeptionsdurchgang wählten wir die Methoden

(1) Unterstreichen: Schüler/innen unterstreichen Wörter im Text,

(2) Schreiben: Schüler/innen schreiben Wörter aus dem Text heraus

(3) Reden: Im Unterrichtsgespräch wird über den Inhalt des Textes geredet

(4) Schüler/innen reden: Schüler/innen reden zu zweit über den Inhalt des Textes (anhand von Leitfragen).

Die Verfahren (1) Unterstreichen und (2) Wörter herausschreiben sind reduktive Verfahren, die geeignet sind, im Sinne der van Dijkschen Makroregeln mentale Textverarbeitungsprozesse $\mathrm{zu}$ stimulieren und eine propositionale Textrepräsentation $\mathrm{zu}$ erstellen. Die Verfahren (3) im Unterrichtsgespräch über den Inhalt des Textes reden und (4) Schüler/innen reden zu zweit über den Inhalt des Textes gehören zu den elaborativen Textverarbeitungsstrategien, bei denen durch Anschlusskommunikation Textstellen akzentuiert und Inferenzen gezogen werden und somit ein situatives Textmodell generiert wird. Mit Ausnahme der Strategie (4) wurden alle Methoden des ersten mit denen des zweiten Rezeptionsdurchgangs kombiniert und getestet, so dass es neun Methodenkombinationen ergab. Die Strategie (4), Schüler/innen reden zu zweit über den Inhalt des Textes, wurde gesondert behandelt. In der 4. Klasse ist dies noch kein eingeübtes Verfahren; es verlangt von den Schüler/innen große Selbständigkeit und Disziplin. Dies kann nicht durchgängig erwartet werden. Dennoch ist es ein Verfahren, das zu den Verfahren "Wörter unterstreichen" und "Wörter herausschreiben" passt, da die Schüler/innen eigenaktiv, ohne die Steuerung durch die Lehrkraft, arbeiten. Wir testeten diese Verfahren nur in einer Methodenkombination, und zwar zusammen mit dem Aktivieren von Vorwissen im Unterrichtsgespräch. Beide Verfahren sind kommunikativ und wir erwarteten, mit dieser Kombination Aufschluss über die Rolle des Redens beim Textverstehen zu erhalten.

Insgesamt ergaben sich zehn Methodenkombinationen (Abb.1), die gleichzeitig zehn Untersuchungsfelder darstellten. Jede Klasse testete nur ein Untersuchungsfeld. Im Anschluss an das Rezipieren des Textes mittels einer bestimmten Methodenkombination wurde ein Lesetest durchgeführt, der in Anlehnung an den Hamburger Lesetest (Lehmann et al. 1997) erstellt wurde. ${ }^{2}$ Die Untersuchung wurde mit 1450 Schüler/innen im Februar 2003 durchgeführt.

\footnotetext{
2 Der Test bestand aus vier Fragen mit je vier Distraktoren, von denen im Multiple-Choice-Verfahren die richtige Antwort anzukreuzen war. Nur eine Antwort war richtig; dies war den Schüler/innen bekannt. Die Antwortmöglichkeiten bestanden aus ganzen Sätzen, so dass textnahes Lesen gefordert war. Genaueres siehe Belgrad/Grütz/Pfaff (2003) und (2004).
} 


\begin{tabular}{|c|c|c|c|}
\hline Stilllesen & M 1 & M 2 & M 3 \\
\hline $\begin{array}{c}\text { 1. Rezeptions- } \\
\text { durchgang }\end{array}$ & Still Lesen & Still Lesen & Still Lesen \\
\hline $\begin{array}{c}\text { 2. Rezeptions- } \\
\text { durchgang }\end{array}$ & $\begin{array}{c}\text { Still Lesen und } \\
\text { Unterstreichen }\end{array}$ & $\begin{array}{c}\text { Still Lesen und } \\
\text { Reden über Text (UG) }\end{array}$ & $\begin{array}{c}\text { Still Lesen und } \\
\text { Schreiben }\end{array}$ \\
\hline
\end{tabular}

\begin{tabular}{|c|c|c|c|}
\hline $\begin{array}{c}\text { Vorlesen } \\
\text { 1. Rezeptions- } \\
\text { durchgang }\end{array}$ & Morlesen & M 5 & M 6 \\
\hline $\begin{array}{c}\text { 2. Rezeptions- } \\
\text { durchgang }\end{array}$ & $\begin{array}{c}\text { Still Lesen und } \\
\text { Unterstreichen }\end{array}$ & $\begin{array}{c}\text { Still Lesen und } \\
\text { Reden über Text }(\mathcal{T} \text { (U) }\end{array}$ & $\begin{array}{c}\text { Still Lesen und } \\
\text { Schreiben }\end{array}$ \\
\hline
\end{tabular}

\begin{tabular}{|c|c|c|c|}
\hline Aktiv.Vorwissen & M 7 & M 8 & M 9 \\
\hline $\begin{array}{c}\text { 1. Rezeptions- } \\
\text { durchgang }\end{array}$ & $\begin{array}{c}\text { Aktivieren von } \\
\text { Vorwissen }\end{array}$ & $\begin{array}{c}\text { Aktivieren von } \\
\text { Vorwissen }\end{array}$ & $\begin{array}{c}\text { Aktivieren von } \\
\text { Vorwissen }\end{array}$ \\
\hline $\begin{array}{c}\text { 2. Rezeptions- } \\
\text { durchgang }\end{array}$ & $\begin{array}{c}\text { Still Lesen und } \\
\text { Unterstreichen }\end{array}$ & $\begin{array}{c}\text { Still Lesen und } \\
\text { Reden über Text }(\mathcal{U})\end{array}$ & $\begin{array}{c}\text { Still Lesen und } \\
\text { Schreiben }\end{array}$ \\
\hline
\end{tabular}

\begin{tabular}{|c|}
\hline M 10 \\
\hline $\begin{array}{c}\text { Aktivieren von } \\
\text { Vorwissen }\end{array}$ \\
\hline $\begin{array}{c}\text { Still Lesen und } \\
\text { Schüler/innen reden } \\
\text { über } \mathcal{T}_{\text {ext }}\end{array}$ \\
\hline
\end{tabular}

Abb. 1: Forschungsdesign: Modell der zehn Methodenkombinationen

Legende: M: Methodenkombination

UG: Unterrichtsgespräch

\subsection{Erwartete Ergebnisse}

Vor dem Hintergrund der Befunde der IGLU-Studie und der PISA-Studie gingen wir von einem generellen besseren Abschneiden der Mädchen aus. Da für unsere Untersuchung die Ergebnisse der IGLU-Studie, deren Proband/innen ebenfalls in der 4. Jahrgangsstufe waren, besonders relevant sind, vermuteten wir analog eine nur geringfügige Überlegenheit der Mädchen. 
Eine gewisse Unwägbarkeit bei der Prognose lag in der Textsorte: In unserer Studie haben wir es mit einem Sachtext mit dem Thema "Wie eine Zeitung entsteht" (Pusteblume. Sachbuch 4) $\mathrm{zu}$ tun. In der IGLU-Studie ist der Geschlechterunterschied beim Lesen von Informationstexten in Deutschland zwar recht gering (vgl. Bos et al. 2003: 114). Doch liegt dem Text themengemäß ein Skript, die Stationen der Zeitungsproduktion, zugrunde. Damit enthält er eine narrative Grundstruktur. Die generelle Überlegenheit der Mädchen bei kontinuierlichen Texten, insbesondere bei narrativen Texten (Elley 1994), ließ daher die Überlegenheit der Mädchen auch bei dem Sachtext der Studie vermuten.

Betrachtet man den geschlechterspezifischen Zugriff auf einzelne Rezeptionsstrategien, wurden folgende Überlegungen angestellt: Den Mädchen werden bessere kommunikative Fähigkeiten im Mündlichen und eine aufgabenorientiertere Gesprächsführung zugeschrieben (z.B. Ruegg 1995; Kotthoff 2003). Es liegt daher die Vermutung nahe, dass im 1. Rezeptionsdurchgang das Verfahren Aktivieren von Vorwissen (Unterrichtsgespräch über das Thema des Textes) und im 2. Rezeptionsdurchgang das Verfahren Reden über den Text zu besseren Ergebnissen bei den Mädchen im Lesetest führen als die anderen Verfahren. Diese Vermutung lässt sich auch durch das Ergebnis der PISA-Studie stützen, dass Mädchen besonders dann besser abschneiden, wenn es darum geht, Textinhalte mit dem eigenen Vorwissen $\mathrm{zu}$ verknüpfen und eigene Erfahrungen einzubringen. Jungen fällt dies offensichtlich schwerer (vgl. Stanat/Kunter: 255, 266). Genau diese Fähigkeiten werden insbesondere bei der Methode des Aktivierens von Vorwissen und in gewissem Maße auch bei der des Reden über den Text verlangt.

Bei den Jungen wurde die Eignung von Rezeptionsstrategien vermutet, die ihnen eine individuell steuerbare Rezeptionsweise ermöglichen. Diesem Bedürfnis kommen die Verfahren des stillen Lesens und des Unterstreichens sowie des Herausschreibens von Wörtern nahe.

\subsection{Ergebnisse}

\subsubsection{Ergebnisse im Vergleich von Mädchen und Jungen}

Die Mädchen sind den Jungen im Leseverstehen des Sachtextes überlegen. Bei einer Punkteskala von 0 - 4 erzielen die Mädchen einen Mittelwert von 2,66 Punkten, die Jungen einen Mittelwert von 2,38 Punkten. Damit sind die Mädchen knapp einen drittel Punkt besser als die Jungen. Bei maximal vier zu erreichenden Punkten ist dies ein deutlich besseres Ergebnis.

Die Mädchen weisen bei fast allen Methodenkombinationen bessere Ergebnisse auf als die Jungen ${ }^{3}$. Folgendes Diagramm (Abb.2) zeigt die Ergebnisse der Methodenkombinationen im Vergleich zwischen Mädchen und den Jungen.

\footnotetext{
3 Bei der Methodenkombination M9 Aktivierung von Vorwissen und Schreiben haben die Jungen ein etwas besseres Ergebnis: Mittelwert: Jungen 2,48 - Mädchen: 2,42. Der Unterschied ist jedoch nicht signifikant.
} 


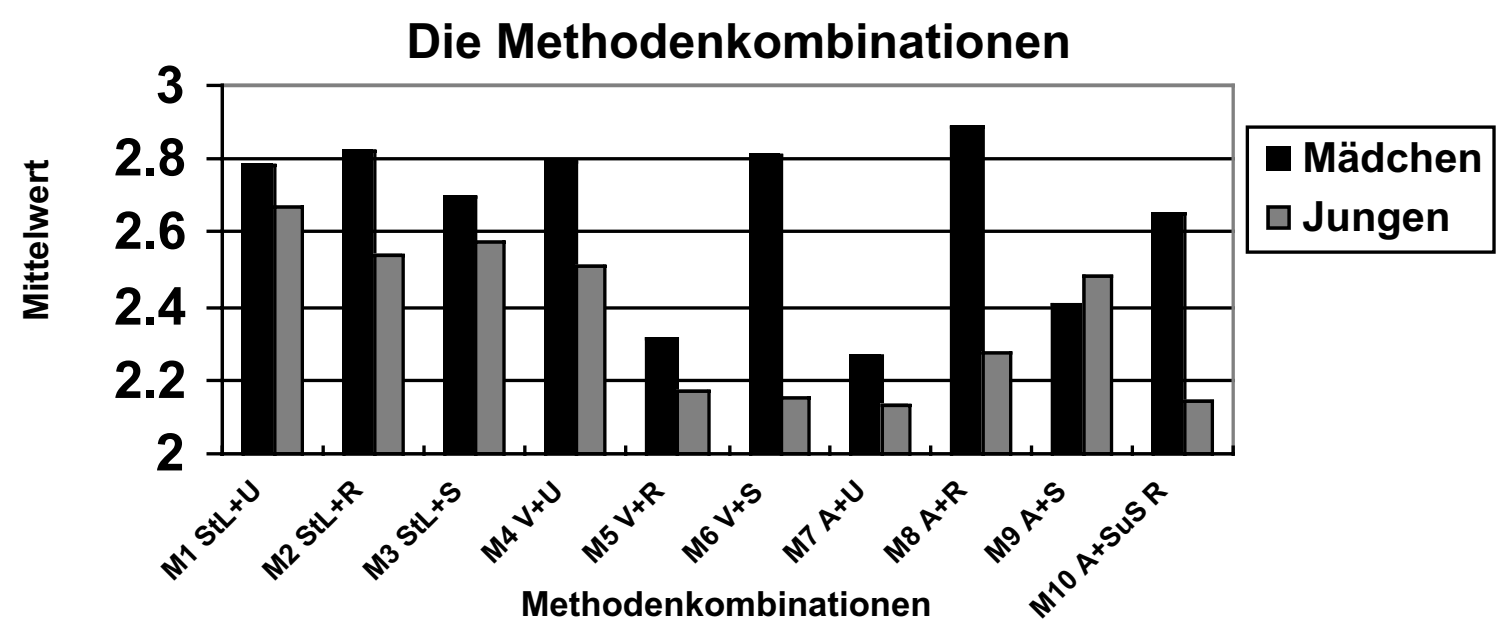

Abb. 2: Ergebnisse der Methodenkombinationen bei Mädchen und Jungen

Ein Vergleich der Methodenkombinationen mit hochsignifikant unterschiedlichen Ergebnissen bei Mädchen und Jungen zeigt folgendes Bild:

- Die Methodenkombination (M6) Vorlesen und Schreiben, bei den Mädchen eine der vier Methodenkombinationen mit ungefähr gleichwertigem zweiten Platz, gehört bei den Jungen zu den Methodenkombinationen mit dem schlechtesten Ergebnis (Mittelwerte: Mädchen 2,81 - Jungen 2,15) .

- Während die Methodenkombination (M8) Aktivierung von Vorwissen und Reden für die Mädchen am besten geeignet ist, liegt diese Methodenkombination bei den Jungen im hinteren Feld (Mittelwerte: Mädchen 2,89 - Jungen 2,27).

- Die Methodenkombinationen (M10) Aktivierung von Vorwissen und Schüler reden liegt bei den Mädchen im Mittelfeld, bei den Jungen gehört sie zu den für das Leseverstehen von Sachtexten ungünstigsten Methoden (Mittelwerte: Mädchen 2,65 - Jungen 2,14) .

Die erwartete Überlegenheit der Mädchen hat sich bestätigt, ebenso die Vermutung, dass die Mädchen bei Kombinationen mit kommunikativen Verfahren besser abschneiden als die Jungen. Bei den Methodenkombination (M8) Aktivierung von Vorwissen und Reden und (M10) Aktivierung von Vorwissen und Schüler reden sind jeweils zwei kommunikative Verfahren beteiligt. Nicht schlüssig ist allerdings, warum Kombinationen, bei denen nur ein kommunikatives Verfahren beteiligt ist, bei Mädchen divergente Ergebnisse aufweisen und warum Mädchen bei der Methodenkombination (M6) Vorlesen und Schreiben so viel besser abschneiden als die Jungen. Zu einer genaueren Interpretation müssen die Ergebnisse nach Geschlechtern getrennt analysiert werden. 


\subsubsection{Ergebnisse bei den Jungen}

Bei den Jungen sind all die Verfahren, die stilles Lesen im 1. Rezeptionsdurchgang haben, am erfolgreichsten. Die Methodenkombination (M1) Stillesen und Unterstreichen schneidet dabei am besten ab. An zweiter Stelle folgt die Kombination (M2) Still lesen und Schreiben, dicht gefolgt von der Methodenkombination (M3) Stilllesen und Reden.

Die beiden anderen Verfahren des 1. Rezeptionsdurchgangs, das Vorlesen und das Aktivieren von Vorwissen im Unterrichtsgespräch, haben zu unterschiedlich verteilte Werte, als dass eine eindeutige Präferenz abgelesen werden könnte. Kombinationen mit diesen Verfahren führen zu mittleren bis schlechten Ergebnissen.

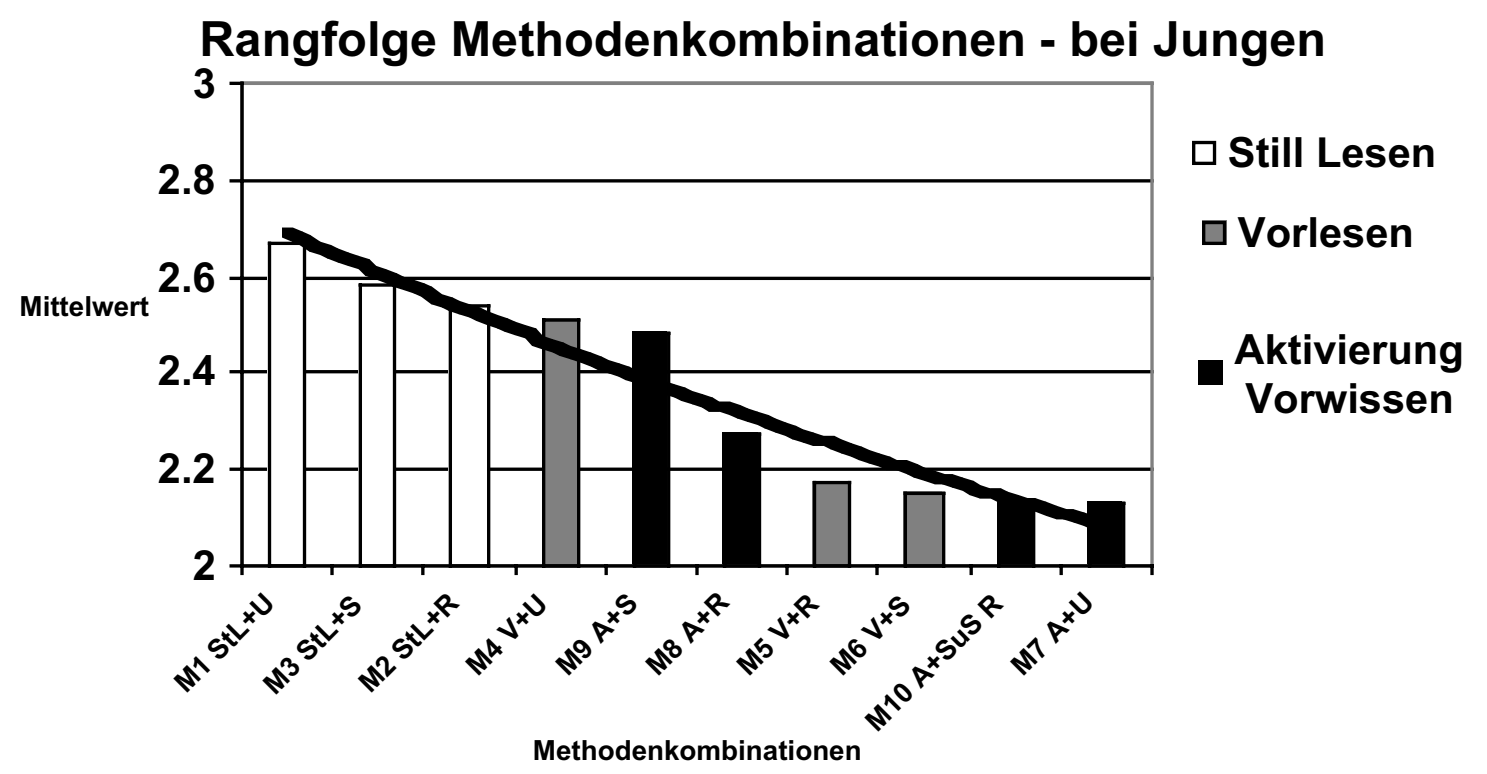

\section{Abb. 3: Rangfolge der Methodenkombinationen bei Jungen}

Bei den Verfahren des 2. Rezeptionsdurchgangs (Unterstreichen, Schreiben, Reden über den Text im Unterrichtsgespräch, Schüler/innen reden über den Text) zeigt sich bei den Jungen eine gewisse Präferenz für das Unterstreichen. Das Unterstreichen ist das Verfahren, das in Kombination mit den Hauptverfahren Stilles Lesen und Vorlesen zu den besten Ergebnissen führt: (M1) Stilllesen und Unterstreichen sowie (M4) Vorlesen und Unterstreichen.

Die Jungen erzielen also die besten Ergebnisse, wenn sie einen Text still durchlesen. Dies kann in zweierlei Hinsicht interpretiert werden:

1) Kognitionspsychologisch: Es ist zu vermuten, dass beim stillen Lesen hauptsächlich Bottom-up-Prozesse wirken: Die Information aus dem Text ist hier ausschlaggebend für das Textverstehen. So scheint sich Perfettis Theorie der verbalen Effizienz zu bestätigen: Die Fähigkeit zu lesen hängt von der Wirksamkeit der hierarchieniedrigen Verarbeitungsprozesse auf Wort- und Satzebene ab. 
2) Kontextbezogen: Offensichtlich brauchen die Jungen Rahmenbedingungen, die es ihnen ermöglichen, in auf den Text fokussierter Konzentration zu arbeiten. Wird ein Text vorgelesen oder wird im Unterrichtsgespräch Vorwissen aktiviert, wirken Dritte in den Rezeptionsprozess ein und behindern diesen unter Umständen.

Unter den textbearbeitenden Strategien des 2. Rezeptionsdurchgangs ist das Unterstreichen das für die Jungen geeignetste Verfahren. Dieses Verfahren passt zum stillen Lesen eines Textes, da es sich auch hier um ein Verfahren mit der Möglichkeit handelt, sich auf den Text zu konzentrieren. Zudem ist dieses Verfahren effizient: Der Gedanke an eine Ökonomie der Rezeption als Prinzip des Lesens drängt sich auf. Unterstreichen ist sicher am wenigsten aufwändig, Schreiben sowie Reden über den Text hingegen am aufwändigsten bzw. am zeitintensivsten oder am wenigsten zielgerichtet (Reden). Diese Interpretation geht durchaus mit dem Befund vieler Studien einher, dass Männer viel zielorientierter lesen als Frauen und die Texte eher überfliegen.

Die Bevorzugung des überfliegenden Lesens könnte auch ein Grund für das schlechtere Abschneiden der Jungen sein. Jungen neigen dazu, Texte oberflächlicher zu lesen. Vergleichszahlen der Studie der Stiftung Lesen von 1992 und 2000 zeigen eine deutliche Zunahme dieser Strategie. Die Aussage " Ich überfliege manchmal die Seiten und lese nur das Interessanteste" bestätigten in der Gruppe der bis zu 19-Jährigen im Jahre 1992 11\%, acht Jahre später waren dies bereits $31 \%$. Dabei gab es einen deutlichen Unterschied zwischen den Geschlechtern: Während diese Aussage im Jahr 1992 nur für $3 \%$ mehr Jungen als Mädchen zutraf, waren es im Jahr 2000 11\% mehr Jungen (Stiftung Lesen 2001:19f.). Der Begriff des "Lesezappings", der für diese Rezeptionsstrategie geprägt wurde, lässt darauf schließen, dass der veränderte Umgang mit dem Fernsehen sich auf das Leseverhalten auswirkt.

\subsubsection{Ergebnisse bei den Mädchen}

Die Mädchen weisen das beste Ergebnis bei der Kombination (M8) Aktivierung von Vorwissen und Reden im Unterrichtsgespräch (hochsignifikant) auf (Mittelwert 2,89).

Gleichwertig gute Ergebnisse (mit Mittelwerten zwischen 2,70 und 2,82) erzielen sie bei allen Kombinationen mit dem stillen Lesen und bei zwei Kombinationen mit dem Vorlesen: (M6) Vorlesen und Schreiben (hochsignifikant) und (M4) Vorlesen und Unterstreichen.

Interessant ist das Ergebnis der Methodenkombination (M10) Aktivierung von Vorwissen und Schüler/innen reden. Die Methode des 2. Rezeptionsverfahrens, Schüler/innen reden, ist ein Verfahren, das selbständiges Arbeiten verlangt und in der 4. Klasse noch nicht zu den eingeübten Methoden gehört. Dennoch erzielen die Mädchen hier ein Ergebnis, das mit zu den guten Ergebnissen gerechnet werden darf (Mittelwert 2,65 bei einer Spannbreite von Mittelwerten zwischen 2, 26 und 2,89).

$\mathrm{Zu}$ den drei schlechtesten Ergebnissen führen die Methodenkombinationen (M9) Aktivieren von Vorwissen und Schreiben (Mittelwert 2,41), (M5) Vorlesen und Reden im Unterrichtsgespräch (Mittelwert 2,31) und (M7) Aktivierung von Vorwissen und Unterstreichen (Mittelwert 2,26). 


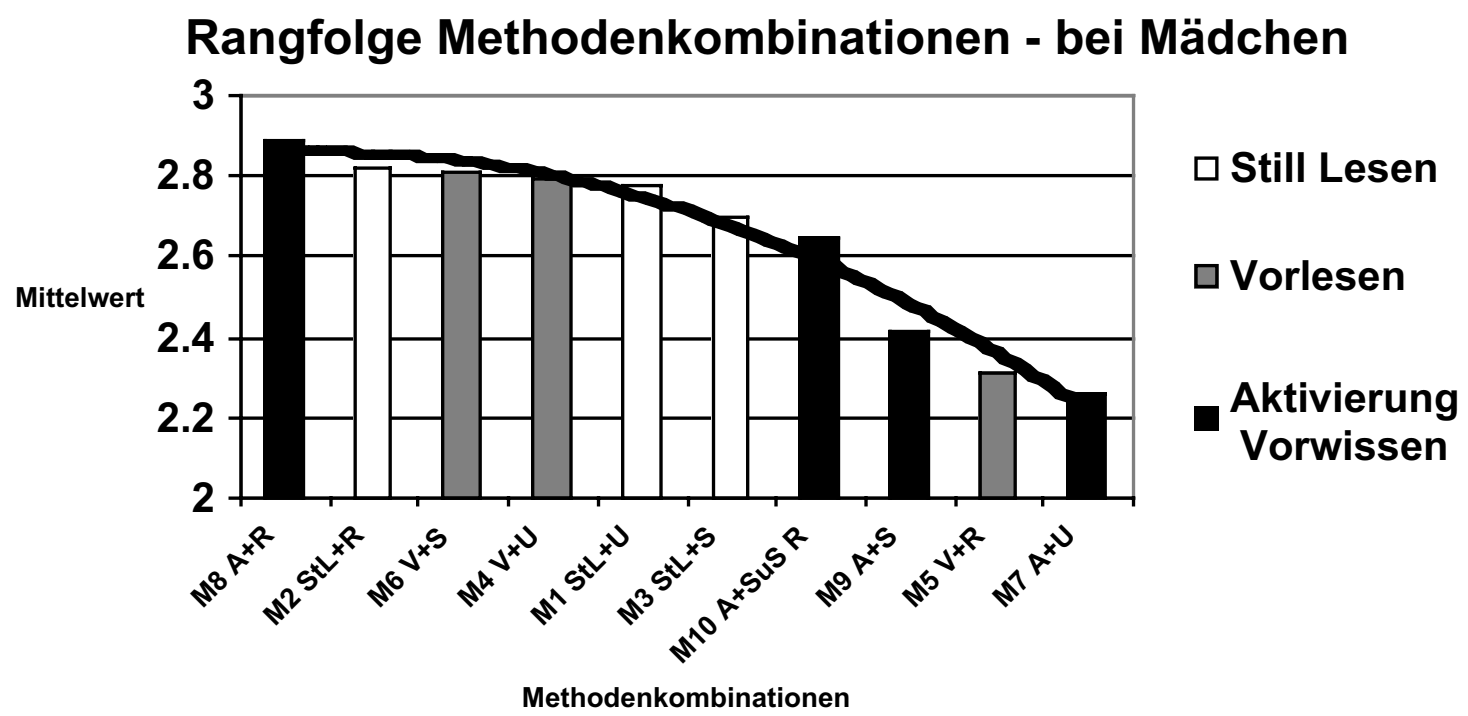

\section{Abb. 4: Rangfolge der Methodenkombinationen bei Mädchen}

Betrachtet man die Methodenkombination nach den Verfahren des 1. Rezeptionsdurchgangs, fällt auf, dass bei den Mädchen kein Verfahren eindeutig als für das Leseverstehen förderlich angesehen werden kann. Das Verfahren Aktivierung von Vorwissen taucht als bestes und als schlechtestes Hauptverfahren auf, ähnlich das Vorlesen, das auf den vorderen Rängen und auf dem vorletzten Platz erscheint. Einzig das Verfahren des Stilllesens zeigt Werte, die relativ nah beieinander im vorderen Bereich liegen (Mittelwerte von 2,82 über 2,78 bis 2,70$)$.

Daraus kann man folgende Schlüsse ziehen:

1) Das Stilllesen ist ein Verfahren, das bei den Mädchen (und bei den Jungen) für das Leseverstehen von Sachtexten geeignet ist.

2) Die Verfahren des Aktivierens von Vorwissen und des Vorlesens sind davon abhängig, wie die Lehrkraft sie ausführt. Beim Aktivieren von Vorwissen ist es die Qualität der Gesprächsführung, beim Vorlesen die Qualität der Präsentation des Textes, die das Textverstehen beeinflusst. Lediglich beim stillen Lesen wird die Leistung ausschließlich von den Schülerinnen (und Schülern) selbst erbracht.

3) Trotz des starken Einflusses der Lehrervariable zeigt sich aber, dass die Geschlechter unterschiedlich reagieren. Immerhin weisen die Mädchen trotz der Unwägbarkeiten des Vorlesens in zwei Kombinationen gute Ergebnisse auf.

Bei den Verfahren des 2. Rezeptionsdurchgangs ist das divergente Abschneiden aller Verfahren festzustellen. Außerdem repräsentiert auch hier ein Verfahren die Extremwerte auf der Rangliste: das Reden. In den Methodenkombinationen Aktivierung von Vorwissen und Reden (M8) und Stilllesen und Reden (M2) liegt es auf den ersten beiden Plätzen. Auch in der Variante Schüler/innen reden erzielt es zusammen mit der Aktivierung von Vorwissen gute Ergebnisse. In Kombination mit dem Vorlesen liegt es hingegen auf dem vorletzten Platz. 
Durch dieses extreme Auseinanderdriften ist es schwierig, die elaborative Rezeptionsstrategie des Redens eindeutig als dem Leseverstehen dienlich zu betrachten. Auch hier könnte die Lehrervariable eine Erklärung für das divergente Abschneiden des Redens sein. Offensichtlich liegt es aber an der Kombination der Verfahren, nicht an einzelnen Verfahren selbst, inwieweit das Verstehen des Sachtextes gefördert wird.

Mit Blick auf die Methodenkombinationen kann konstatiert werden:

1) Mädchen weisen bei sieben von zehn Methodenkombinationen gute Ergebnisse auf. Nur drei Methodenkombination fallen im Ergebnis deutlich ab. Sie sind also generell gute Leserinnen.

2) Die Kombination Aktivierung von Vorwissen und Reden (Unterrichtsgespräch) ist bei Mädchen optimal für das Verstehen von Sachtexten. Dies ist eine Methodenkombination mit zwei kommunikativen Verfahren. Zweimal findet ein Unterrichtsgespräch statt: vor und nach dem Lesen des Textes. Es liegen zwei ähnliche Rezeptionsstrategien vor. Kommunikation stützt bei den Mädchen (im Gegensatz zu den Jungen) den Textverstehensprozess. Dies zeigt sich auch bei Kombinationen mit dem Hauptverfahren Stillesen. Hier ist es die Kombination Stilllesen und Reden, die bei den Mädchen am besten abschneidet. Die beiden anderen Kombinationen mit dem Stilllesen zeigen etwas schlechtere Ergebnisse.

Diese Feststellungen führen zu folgenden Überlegungen: Unter den Mädchen kristallisieren sich bestimmte "Lesetypen" heraus. Unter vorsichtiger Interpretation der Ergebnisse zeigt sich, dass bei der Kombination von kognitiv gleichartigen Strategien eher gute, bei der Kombination von kognitiv unterschiedlichen Strategien eher schlechte Ergebnisse erzielt werden.

- Unter der Annahme, dass im 1. Rezeptionsdurchgang das stille Lesen und das Vorlesen Methoden sind, die eher Bottom-up-Prozesse initiieren und unter der weiteren Annahme, dass im 2. Rezeptionsdurchgang das Unterstreichen und das Herausschreiben von Wörtern aus dem Text reduktive Textverarbeitungsstrategien sind, die im interaktionalen Modell vorwiegend datengesteuert sind, weisen diese Methoden eine genuine Zusammengehörigkeit bei der kognitiven Verarbeitung auf.

- Ähnlich verhält sich dies mit den Kombinationen kommunikativer Verfahren: Die Strategie des 1.Rezeptionsdurchgangs Aktivieren von Vorwissen im Unterrichtsgespräch initiiert lesergesteuert hierarchiehohe Verarbeitungsprozesse; die elaborativen Strategien des 2. Rezeptionsdurchgangs Reden über den Text im Unterrichtsgespräch und Schüler/innen reden über den Text sind Verfahren, bei denen durch die verbale Interaktion Inhalte akzentuiert und Inferenzen gezogen werden und somit die mentale Textbasis eine Veränderung erfährt. Auch diese Verfahren gehören bezüglich der Hierarchieebene und der Art des Verarbeitungsprozesses genuin zusammen.

- Verfahren, die im Sinne der kognitiven Verarbeitung nicht zusammenpassen, zeigen schlechte Ergebnisse: Dies sind die Methodenkombinationen Aktivierung von Vorwissen und Unterstreichen, Aktivierung von Vorwissen und Schreiben sowie Vorlesen und Reden. Bei den 
Methodenkombinationen ist jeweils ein kommunikatives, vorwissens- bzw. wissensgeleitetes Verfahren und ein stärker an den Daten des Textes orientiertes Verfahren beteiligt.

- Eine Sonderstellung nimmt das stille Lesen eines Textes ein: Dass das Stilllesen unabhängig von der Kombination mit einer anderen Methode gute Leseleistungen hervorbringt, könnte folgende Erklärung haben: Das stille Lesen eines Textes bezieht sich zwar vornehmlich auf den Bottom-up-Prozess, verhält sich aber offensichtlich neutral zur Kombination mit anderen Verfahren. Der Einstieg durch das stille Lesen lässt weitere Verarbeitungswege gleichrangig offen.

Die Ergebnisse der Studie legen bei den Mädchen nahe, dass es bestimmte "Lesetypen" gibt, die bei der mentalen Verarbeitung des Textes entweder vorwiegend kommunikative vorwissensgeleitete und elaborative Methoden, und solche, die eher textbasierte und reduktive Methoden bevorzugen. Es kann also von dem eher kommunikationsorientierten und dem eher textorientierten Lese- bzw. Rezeptionstyp ausgegangen werden.

\subsubsection{Bedeutung der Überlegung vom "Lesetyp" für beide Geschlechter}

Die Folgerung aus den Ergebnissen der Studie, dass es bei den Mädchen bestimmte "Lesetypen" gibt, müsste in weiteren Studien gezielt überprüft werden. Zu fragen ist auch, ob sich solche Lesetypen nicht auch bei den Jungen herauskristallisieren. Zwar ist das konsistent gute Abschneiden des Stilllesens bei den Jungen ein überwältigend eindeutiger Befund und kognitionspsychologisch zu würdigen, doch sollte man überlegen, ob nicht auch andere Verfahren einzeln oder in bestimmter Kombination zum Erfolg führen könnten, wenn sie nur richtig zum Zuge kämen. (Zu denken ist an die Beobachtung, dass lernwillige Jungen sich häufig erst gegen ihre Mitschüler durchsetzen müssen, um lernen zu können.)

Denkbar ist aber auch ein Zusammenhang zwischen Leseintensität und der Bevorzugung bestimmter Lesestrategien. So stellt sich die Frage, ob z.B. bei Vielleserinnen und -lesern eher hierarchiehohe Verarbeitungsprozesse in Gang gesetzt werden, die im strategischen Bereich liegen (Aktivierung von Vorwissen), bei Wenigleserinnen und -lesern hingegen eher textbasierte Verarbeitungsprozesse, die eher automatisiert ablaufen. Aus Studien zu Leseaktivitäten ist bekannt, dass Jungen weniger lesen als Mädchen (z.B. Bertschi-Kaufmann 2002:152) und weniger gute Leseleistungen erbringen als Mädchen. In der vorliegenden Untersuchung erzielen die Jungen die besten Leseleistungen beim stillen Lesen eines Textes, wobei im 2. Rezeptionsdurchgang das Unterstreichen zu besseren, das Reden über den Text zu schlechteren Ergebnissen führt. Das weist sie als eher textorientierten Lesetyp aus. Es könnte also ein Zusammenhang bestehen zwischen Leseintensität, Leseleistung und Lesetyp. Diese Vermutung bestätigt sich auch durch eine weitere Korrelation in der Studie: Schüler/innen mit Migrationshintergrund, die an sich größere sprachliche Schwierigkeiten haben als Muttersprachler/innen, weisen im Durchschnitt schlechtere Leseleistungen auf. Sie bevorzugen aber die gleichen Lesestrategien wie die Jungen (Belgrad/Grütz/Pfaff 2004).

Ob nun auch bei Mädchen der Zusammenhang zwischen Leseintensität und Lesetyp existiert, kann hier nicht beantwortet werden. In der Studie wurde nicht erhoben, ob die Schüler/innen 
viel oder wenig lesen. Die überwiegend guten Leseleistungen der Mädchen bei bestimmten Methodenkombinationen lassen lediglich den Schluss von der Bevorzugung bestimmter Lesestrategien und der Ausprägung bestimmter Lesetypen zu.

\subsection{Zusammenfassung der Ergebnisse}

Die Ergebnisse der Studie wurden in diesem Kapitel entsprechend der eingangs dargelegten Leseverstehenstheorien vorwiegend aus kognitionspsychologischer Sicht interpretiert. Berücksichtigt wurden z.T. auch die im ersten Kapitel dargelegten Befunde zu geschlechtsspezifischen Verhalten, das sich auf das schulische Verhalten im Allgemeinen und auf das Rezipieren von Texten im Besonderen auswirkt. Eine kurze Zusammenfassung soll die wichtigsten Ergebnisse der Studie ins Blickfeld rücken.

- In der 4. Jahrgangstufe sind die Mädchen den Jungen beim Verstehen von Sachtexten überlegen. Sie bringen im Lesetest im Durchschnitt bessere Leistungen als die Jungen.

- Die größten Unterschiede bei der Leseleistung von Mädchen und Jungen treten bei folgenden Methodenkombinationen auf:

Aktivieren von Vorwissen und Reden

Aktivieren von Vorwissen und Schüler reden

Vorlesen und Schreiben.

Die Leseleistungen der Mädchen zeigen hier sehr gute bis gute Ergebnisse. Bei den Jungen führen diese Methodenkombinationen hingegen zu den schlechtesten Ergebnissen. Anhand der beiden erstgenannten Methodenkombinationen wird offenkundig, dass die Mädchen deutlich besser als die Jungen mit Verfahren umgehen können, bei denen es auf den verbalen Austausch ankommt.

- Bei den Jungen führen alle Methodenkombinationen mit dem Stilllesen als Verfahren des 1. Rezeptionsdurchgangs zu den besten Ergebnissen. Bei den Verfahren des 2. Rezeptionsdurchgangs zeigt sich eine tendenzielle Präferenz für das Unterstreichen.

- Bei den Mädchen führt die Methodenkombination Aktivieren von Vorwissen und Reden zum besten Ergebnis. Unter den Verfahren des 1. Rezeptionsdurchgangs kann kein Verfahren eindeutig als erfolgreiches Verfahren benannt werden. Bei den Verfahren des 2. Rezeptionsdurchgangs zeigt sich eine tendenzielle Präferenz für das Reden. Der Leseerfolg hängt von der Kombination der Verfahren ab. Es kristallisieren sich zwei Lesetypen heraus: der kommunikationsorientierte Lesetyp und der textorientierte Lesetyp.

- Das stille Lesen ist eine Methode, die bei beiden Geschlechtern das Verstehen von Sachtexten fördert. Daraus ist zu schließen, dass dieses Verfahren eine für das Rezipieren von Sachtexten genuine Methode ist.

Die vorliegenden Befunde über den geschlechterabhängigen Erfolg bzw. Misserfolg von Lesestrategien bei Sachtexten in der Grundschule verlangen nach Antworten bezüglich ihrer didaktischen Umsetzung im Unterricht im Sinne einer gezielten geschlechterspezifischen Leseförderung. Dabei ist zu berücksichtigen, dass die Befunde anhand eines kontinuierlichen Sachtextes mit narrativer Struktur gewonnen wurden. Eine allgemeine Gültigkeit der 
erfolgreichen Anwendung der jeweils präferierten Lesestrategien auf jedwede Ausprägung von Sachtexten, insbesondere auf diskontinuierliche Texte, die seit der PISA-Studie mehr und mehr ins Bewusstsein rücken, kann nicht ohne weiteres unterstellt werden. Dennoch liefert die Untersuchung wichtige Anhaltspunkte für den Unterricht und für weitere Studien zur Leseforschung.

\section{Literaturangaben}

Alphen, Ingrid C. van (1996): "Wie die Jungen das Lachen lernten - und wie es den Mädchen wieder verging". In: Kotthoff, Helga (ed.): Das Gelächter der Geschlechter. Konstanz: 217-227.

Artelt, Cordula/Stanat, Petra/Schneider, Wolfgang/ Schiefele, Ulrich (2001): "Lesekompetenz: Testkonzeption und Ergebnisse". In: Baumert et al. (eds.): 69-137.

Baumert, Jürgen /Klieme, Eckhard/Neubrand, Michael/Prenzel, Manfred /Schiefele, Ulrich/Schneider, Wolfgang/Stanat, Petra/Tillmann, Klaus-Jürgen/Weiß, Manfred (eds.) (2001): PISA 2000. Basiskompetenzen von Schülerinnen und Schülern im internationalen Vergleich. Opladen.

Baumert, Jürgen/Artelt, Cordula/Klieme, Eckhard/Neubrand, Michael/Prenzel, Manfred/ Schiefele, Ulrich/Schneider, Wolfgang/Tillmann, Klaus-Jürgen/Weiß, Manfred (eds.) (2003): PISA 2000 - Ein differenzierter Blick auf die Länder der Bundesrepublik Deutschland. Opladen.

Baron-Cohen, Simon (2004): "Vom ersten Tag an anders. Das weibliche und das männliche Gehirn". Psychologie heute, März 2004: 44-47.

Belgrad, Jürgen/Grütz, Doris/Pfaff, Harald (2003): Forschungsbericht 2003. Studie zum Verstehen von Sachtexten in der Grundschule 4. Klasse. Pädagogische Hochschule Weingarten.

Belgrad, Jürgen/Grütz, Doris/Pfaff, Harald (2004): "Verstehen von Sachtexten. Eine Studie in der Grundschule 4. Klasse". Didaktik Deutsch 17: 26-43.

Bertschi-Kaufmann, Andrea (2002a): "Multimedia und Leseförderung in der Schule. Ergebnisse aus den Forschungsprojekten 'Literalität im medialen Umfeld' und 'Lesen im Kontext neuer Medien'". In: Bonfadelli, Heinz/Bucher, Priska (eds): Lesen in der Mediengesellschaft. Stand und Perspektive der Forschung. Zürich: 148-161.

Bertschi-Kaufmann, Andrea (2002b): "Lesen und Schreiben in einer Medienumgebung. Empirisches Arbeiten im Raum Schule". In: Kammler, Clemens/Knapp, Werner (eds.): 141-155.

Bischoff, Ulrike/Heidtmann, Horst (2002a): Leseverhalten in der Erlebnisgesellschaft. Eine Untersuchung zu den Leseinteressen und Lektüregratifikationen von Jungen. Weinheim/ München: 241-267.

Bischoff, Ulrike/Heidtmann, Horst (2002b): "Lesen Jungen ander(e)s als Mädchen? Untersuchungen zu Leseinteresse und Lektüregratifikationen". medien praktisch. Zeitschrift für Medienpädagogik 3/02 Nr. 103: 27-31.

Bonfadelli, Heinz/Bucher, Priska (ed.) (2002): Lesen in der Mediengesellschaft. Stand und Perspektive der Forschung. Zürich. 
Bonfadelli, Heinz/Fritz, Angela (1993): "Lesen im Alltag von Jugendlichen". In: Bonfadelli, Heinz/Fritz, Angela/Köcher, Renate (eds.): 7-213.

Bonfadelli, Heinz/Fritz, Angela/Köcher, Renate (1993): Lesesozialisation. Bd. 2 . Leseerfahrungen und Lesekarrieren: Studien der Bertelsmann Stiftung. Gütersloh.

Bos, Wilfried/Lankes, Eva-Maria/Prenzel, Manfred/Schwippert, Kurt/Walther, Gerd/Valtin, Renate (eds) (2003): Erste Ergebnisse aus IGLU. Schülerleistungen am Ende der vierten Jahrgangsstufe im internationalen Vergleich. Münster/New York/München/Berlin.

Brügelmann, Hans/Balhorn, Heiko/Füssenich, Iris (eds.) (1995): Am Rande der Schrift. Zwischen Sprachenvielfalt und Analphabetismus. Lengwil.

Christmann, Ursula/Groeben, Norbert (2002): "Anforderungen und Einflussfaktoren bei Sachund Informationstexten". In: Groeben, Norbert/Hurrelmann, Bettina (eds.): 150-173.

Elley, Warwick Barham (ed.) (1994): The IEA Study of Reading Literacy: Achievement and instruction in thirty-two school systems. Oxford, UK.

Franz, Kurt/Franzmann, Bodo/Payrhuber, Franz-Josef/Schön, Erich (1999): "Muß-Lektüre versus Lust-Lektüre?" In: Groeben, Norbert (ed.): 78-88.

Fuchs, Claudia (2001): Barbie trifft He-Man. Kinder erzählen über ihre Spielwelten und ihre Alltagswelt. Freiburg i. Brsg.

Garbe, Christine (2003a): "Warum lesen Mädchen besser als Jungen? Zur Notwendigkeit einer geschlechterdifferenzierenden Leseforschung und Leseförderung." In: Abraham, Ulf et al. (eds.): Deutschdidaktik und Deutschunterricht nach PISA. Freiburg i. Brsg.: 69-89.

Garbe, Christine (2003b): "Mädchen lesen ander(e)s". JuLit 2/03: 14-29.

Groeben, Norbert (ed.) (1999): Lesesozialisation in der Mediengesellschaft. Internationales Archiv für Sozialgeschichte der deutschen Literatur 10. Sonderheft. Tübingen.

Groeben, Norbert/Hurrelmann, Bettina (eds.) (2002): Lesekompetenz - Bedingungen, Dimensionen, Funktionen. Weinheim/München.

Hurrelmann, Bettina (2002): "Prototypische Merkmale der Lesekompetenz". In: Groeben, Norbert/Hurrelmann, Bettina. (eds.): 275-286.

Groeben, Norbert (ed.) (1999): Lesesozialisation in der Mediengesellschaft. Internationales Archiv für Sozialgeschichte der deutschen Literatur 10. Sonderheft. Tübingen.

Hamm, Ingrid/Langen, Claudia (2002): "Das Lesebarometer - Lesen und Umgang mit Büchern in Deutschland". In: Bonfadelli, Heinz/Bucher, Priska (eds.): Lesen in der Mediengesellschaft. Stand und Perspektive der Forschung. Zürich: 43-56.

Harmgarth, Friederike (ed.) (1999): Das Lesebarometer - Lesen und Umgang mit Büchern in Deutschland. Gütersloh.

Hentschel, Ute (2000): "Bücher für Bruno. Neue Jungen im Bilder-, Kinder- und Jugendbuch?". Eselsohr 3: 7.

Hurrelmann, Bettina (2002): "Leseleistung - Lesekompetenz. Folgerungen aus PISA, mit einem Plädoyer für ein didaktisches Konzept des Lesens als kultureller Praxis". Praxis Deutsch 176: 6-18.

Hurrelmann, Bettina/Hammer/Nieß (1993): Lesesozialisation. Bd.1. Leseklima in der Familie: eine Studie der Bertelsmann Stiftung. Gütersloh. 
Kessels, Ursula (2004): "Mädchenfächer - Jungenfächer? Geschlechtertrennung im Unterricht". In: Becker, Gerold et al. (eds.): Heterogenität. Unterschiede nutzen Gemeinsamkeiten stärken. Friedrich Jahresheft XXII 2004: 90-94.

Köcher, Renate (1993): "Lesekarrieren - Kontinuität und Brüche". In: Bonfadelli, Heinz/ Fritz, Angela/Köcher, Renate (1993): Lesesozialisation. Bd.2. Leseerfahrungen und Lesekarrieren: Studien der Bertelsmann Stiftung. Gütersloh: 215-310.

Kotthoff, Helga (2003): "Problemgruppe Jungen? Neue Fragen für die Kinder- und Jugendforschung, Ethnografie der Schule und die Gesprächsanalyse". Der Deutschunterricht 2/2003: 85-88.

Lehmann, Rainer H. (1994). "Lesen Mädchen wirklich besser? Ergebnisse aus der internationalen IEA-Lesestudie". In: Richter Sigrund/Brügelmann, Hans (eds.): Mädchen lernen ANDERS lernen Jungen: Geschlechtsspezifische Unterschiede beim Schriftspracherwerb. Konstanz: 99-109.

Lehmann, Reiner H./ Peek, Rainer/Poerschke, Jan (1997): HAMLET 3-4. Hamburger Lesetest für 3. und 4. Klassen. Weinheim/Basel.

Linke, Angelika/Oomen-Welke, Ingelore (eds.) (1995): Herkunft, Geschlecht und Deutschunterricht: oben - unten, von hier - von anderswo, männlich - weiblich. Freiburg i. Brsg.

Nickel-Bacon, Irmgard (2003): "Hat Lesen ein Geschlecht?". Schüler 2003. Lesen + Schreiben: $30-31$.

Oakhill, Jane/Garnham, Alan (1988): Becoming a skilled reader. New York.

Oerter, Rolf (1999): "Theorien der Lesesozialisation - Zur Ontogenese des Lesens". In: Groeben, Norbert (ed.): Lesesozialisation in der Mediengesellschaft. Internationales Archiv für Sozialgeschichte der deutschen Literatur 10. Sonderheft. Tübingen: 27-55.

Perfetti Charles A. (1985): Reading ability. New York.

Pusteblume Sachbuch 4 (1998): Text: Wie eine Zeitung entsteht. Hannover: 66f.

Richter, Sigrun (1999): "Mädchen und Jungeninteressen beim Schreiben und Lesen". Grundschule 12/1999: 38-40.

Richter, Sigrun/Brügelmann, Hans (eds.) (1994): Mädchen lernen anders - anders lernen Jungen: Geschlechtsspezifische Unterschiede beim Schriftspracherwerb. Lengwil.

Richter, Tobias/Christmann, Ursula (2002): "Lesekompetenz: Prozessebenen und interindividuelle Unterschiede". In: Groeben, Norbert/ Hurrelmann, Bettina (eds.): 25-58.

Richter, Karin/Plath, Monika (2002): "Die Bedeutung der Entwicklung von Lesemotivation in der Grundschule". In: Franz, Kurt/Payrhuber, Franz-Josef (eds): Lesen heute. Leseverhalten und Leseförderung im Kontext der PISA-Studie. Baltmannsweiler: 41-58.

Rüegg, Regula (1995): "Stille Mädchen - laute Buben?" In: Linke, Angelika/Oomen-Welke, Ingelore (eds.): 171-188.

Rumelhart, David E. (1975): "Notes on a schema for stories". In: Bobrow, Daniel G./Collins, Alan (eds.): Representation and understanding. New York: 211-236.

Rumelhart, David E. (1994): "Toward an interactive model of reading". In: Singer, Harry (ed.): Theoretical models and processes of reading. Newark Delaware: 864-894. 
Schilcher, Anita (2003): "Geschlechterdifferenzierender Deutschunterricht. Was machen die Jungs? Geschlechterdifferenzierender Deutschunterricht nach PISA". In: Abraham, Ulf/Bremerich-Vos, Albert/Frederking, Volker/Wieler, Petra (eds.): Deutschdidaktik und Deutschunterricht nach PISA. Freiburg i. Brsg.: 361-380.

Spiro, Rand J. (1980): "Constructive processes in prose comprehension and recall". In: Spiro, Rand.J./Bruce, Bertram.C./ Brewer, William F.(eds.): Theoretical issues in reading comprehension: Perspectives from cognitive psychology, linguistics, artificial intelligence and education. Hillsdale, NY: 245-278.

Stanat, Petra/Kunter, Mareike (2001): "Geschlechterunterschiede in Basiskompetenzen". In: Baumert, Jürgen/Klieme, Eckhard/Neubrand, Michael/Prenzel, Manfred/Schiefele, Ulrich/Schneider, Wolfgang/Stanat, Petra/Tillmann, Klaus-Jürgen/Weiß, Manfred (eds.): PISA 2000. Basiskompetenzen von Schülerinnen und Schülern im internationalen Vergleich. Opladen: 249-269.

Stanat, Petra et al. (2003): "PISA und PISA-E: Zusammenfassung der bereits vorliegenden Befunde". In: Deutsches PISA-Konsortium (ed.): PISA 2000. Ein differenzierter Blick auf die Länder der Bundesrepublik Deutschland. Opladen: 51-75.

Stanovich, Keith E.(1980): "Toward an interactive-compensatory model of individual differences in the developpement of reading fluency". Reading Research Quaterly 16: 3271.

Stiftung Lesen (ed.) (2001): Leseverhalten in Deutschland im neuen Jahrtausend. Schriftenreihe "Lesewelten". Bd. 3. Mainz.

Thimm, Katja (2004): "Angeknackste Helden". Der Spiegel, Heft 21/2004: 82-95.

Van Dijk T.A./Kintsch, Walter. (1983): Strategies of discourse comprehension. New York.

Van Kraayenoord, Christina E./Schneider, Wolfgang (1999): "Reading achievement, metacognition, reading self-concept and interest. A study of german students in grade 3 and 4". European Journal of psychology and Education 14: 305-324.

Yekovich, Frank R/Walker, Carol H./Blackmann, Harold S. (1979): "The role of presupposed and focal information in integrating sentences". Journal of Verbal Learning and Verbal Behaviour 18: 535-548. 